\section{$\underset{\substack{\text { hommes } \\ \text { \& migrations }}}{ }$}

\section{Hommes \& migrations}

Revue française de référence sur les dynamiques

migratoires

\section{8 | 2014}

Les Paris des migrants

\title{
Le touriste, le migrant et la fable cosmopolite
}

Mettre en tourisme les présences migratoires à Paris

\section{Amandine Chapuis et Sébastien Jacquot}

\section{(2) OpenEdition}

1 Journals

\section{Édition électronique}

URL : http://journals.openedition.org/hommesmigrations/2999

DOI : 10.4000/hommesmigrations.2999

ISSN : 2262-3353

Éditeur

Musée national de l'histoire de l'immigration

\section{Édition imprimée}

Date de publication : 1 octobre 2014

Pagination : 75-84

ISBN : 978-2-919040-29-2

ISSN : 1142-852X

Référence électronique

Amandine Chapuis et Sébastien Jacquot, «Le touriste, le migrant et la fable cosmopolite », Hommes \& migrations [En ligne], 1308 | 2014, mis en ligne le 01 octobre 2017, consulté le 30 avril 2019. URL :

http://journals.openedition.org/hommesmigrations/2999; DOI : 10.4000/hommesmigrations.2999 


\title{
LE TOURISTE, \\ LE MIGRANT ET LA FABLE COSMOPOLITE \\ METTRE EN TOURISME LES PRÉSENCES MIGRATOIRES À PARIS
}

par AMANDINE CHAPUIS, chercheure post-doc, université Paris-Est, et SÉBASTIEN JACQUOT, maître de conférences, université Paris-I.

\author{
À l'instar des grandes métropoles cosmopolites comme \\ Londres, New York ou Montréal, Paris commence à inscrire ses \\ quartiers de migration dans ses parcours touristiques. \\ À grand renfort de communication institutionnelle, \\ les itinéraires proposés se diversifient, conduisant les touristes \\ à la rencontre des populations immigrées de la capitale. \\ Face à des visites nourries de clichés exotiques, des associations \\ tentent de se réapproprier l'histoire de cette présence \\ migratoire et la mise en tourisme de ces quartiers.
}

"Et vous pensiez que tout était affaire de bérets, de baguettes et de bistros... ? Pour sûr Paris est et restera française. Mais Paris ville-monde vibre aujourd'hui à un rythme bien plus international, celui d'une ville diverse, dynamique et multiculturelle." Le guide Lonely Planet décrit désormais en ces termes l'une des toutes premières destinations touristiques mondiales, rompant avec les stéréotypes de l'imaginaire touristique parisien. À l'heure où triomphe l'idée de la métropole créative ${ }^{1}$, forte de sa diversité culturelle, nombreuses sont les villes qui mettent en avant le dynamisme de leurs communautés migratoires comme témoignages de la vitalité de leur insertion au cour des réseaux économiques et culturels de la mondialisation. Londres, New York, Sydney,
Montréal, Singapour n'hésitent pas à faire de leurs “quartiers ethniques" relookés les symboles matériels de ce nouveau cosmopolitisme et les pierres angulaires d'un marketing urbain visant à attirer les touristes internationaux tant convoités.

Qu'en est-il à Paris, où les imaginaires mobilisés par les acteurs du tourisme restent généralement très classiques, et où plus globalement les questions migratoires demeurent vives dans la société française?

Nous considérons le tourisme non pas uniquement comme l'activité économique d'un secteur bien encadré et florissant de la métropole parisienne (47 millions de touristes en 2013 pour le Grand Paris), mais plutôt comme un ensemble de 
phénomènes participant d'une mise en récit d'éléments culturels. Ces phénomènes impliquent des acteurs divers (entreprises, associations, collectivités locales...) et s'adressent à des groupes très hétérogènes, amalgamés sous la bannière "touristes", qui peuvent être des touristes internationaux ou provinciaux, mais aussi des Parisiens ou Franciliens souhaitant porter un autre regard sur leurs espaces de vie quotidienne. Le quartier, présenté Nous cherchons donc à comcomme "ethnique", constitue un Ailleurs tout comme l'immigré constitue un Autre qui n'est pas assimilable à la figure normative du Parisien, mais ajoute une touche d'exotisme à la destination. prendre ce que cette relation entre tourisme et migrations nous dit de l'activité touristique, ainsi que de celles et de ceux qui sont associé aux migrations dans la métropole parisienne. Le Paris touristique est également fait par ses immigrés en tant que personnages et signifiants de cette fable cosmopolite, dans tout ce qu'elle comporte d'ambiguités politiques, et aussi en tant que coproducteurs. Questionner la relation entre tourisme et migrations à Paris, c'est, pour nous, s'interroger sur ce que la mise en tourisme du fait migratoire dit de l'ancrage des immigrés dans la ville, de leur place politique et de la présentation de leur histoire personnelle et collective avec Paris.

\section{Représenter les quartiers d'immigration}

La visite de certains quartiers d'immigration est devenue incontournable dans beaucoup de métropoles mondiales. Les quartiers "ethniques" à Manhattan de Chinatown et Little Italy sont en tête des guides de New York. Si le discours touristique sur cette ville s'est en grande partie construit autour de l'idée d'une ville-monde composée de multiples eth- noscapes ${ }^{2}$, ce n'est pas le cas de Paris, dont les imaginaires promus restent ceux d'une capitale authentique de la culture française.

Visiter les quartiers d'immigration à Paris revient à adopter des pratiques et des itinéraires marginaux. Les arrondissements aux plus forts taux de populations immigrées, tout comme les communes périphériques, ne correspondent pas aux espaces touristiques les plus fréquentés du Central Tourist District $^{3}$. Cette relation en creux recoupe celle de la moindre fréquentation des quartiers les plus populaires, délaissés par l'activité touristique au profit des quartiers bourgeois qui concentrent les sites culturels et le bâti monumental, dès le XIX siècle ${ }^{4}$. Certains quartiers d'immigration, notamment en proche banlieue, font l'objet d'un déficit flagrant d'attractivité touristique au regard de leurs ressources patrimoniales. La basilique de SaintDenis, berceau de l'art gothique et nécropole des rois de France, accueillait seulement 172121 visiteurs en $2012^{5}$. Saint-Denis est la dernière recommandation du guide Lonely Planet de Paris, à la fin de la rubrique "Beyond central Paris", témoignage aussi de la stigmatisation dont la première couronne francilienne a pu faire l'objet dans les médias internationaux cette dernière décennie, suite aux émeutes de 2005.

Cependant, même si les quartiers migratoires parisiens sont loin de rivaliser avec la renommée de leurs homologues états-uniens, plusieurs sont mentionnés à la fois par les guides touristiques internationaux et par la communication institutionnelle - l'Office du tourisme et des congrès de Paris (OTCP) ou le Comité régional du tourisme d'Île-de-France (CRT) - et attirent indéniablement des flux touristiques. C'est le cas du quartier asiatique du XIII arrondissement, désigné comme le Chinatown parisien : "Furetez de restaurant chinois en échoppe vietnamienne dans le Chinatown de la capitale et vous aurez la sensation d'avoir impercepti- 
blement changé de continent." Le passage Brady est mentionné comme quartier "indien", "passage couvert ancien [qui] pourrait tout aussi bien se trouver à Calcutta”. La Goutte-d'Or est évoquée comme le quartier "nord-africain": "La rue Myrha est la frontière entre l'Afrique centrale et de l'Ouest et le Maghreb, la musique raï laisse rapidement la place au bikutsi camerounais et au mbalax sénégalais ${ }^{6}$."

\section{Faire l'expérience d'un Ailleurs enchanté}

Ces présentations du Paris migratoire par l'édition ou les institutions locales taisent l'expérience des migrants, centrant leur discours sur l'expérience multisensorielle du visiteur qui, au contact de ces lieux "hauts en couleur", aux "effluves d'encens et de curry", accomplit un "voyage sensoriel, visuel et aromatique $e^{8 ”}$. Elles s'appuient sur les "traces" matérielles (enseignes, saris dans les devantures des boutiques...) ou intangibles (odeurs d'épices, langues entendues dans la rue, sonorités musicales s'échappant des cafés...) du peuplement migratoire et de l'ancrage des diasporas dans l'espace urbain. Ces traces sont disséminées et présentées comme pittoresques, cachées, insolites. Bref, le registre de l'exotisme domine la mise en récit de l'immigration à Paris. Il s'appuie avant tout sur les traditions culinaires : les images d'ingrédients et de plats exotiques (mangues, currys, couscous ou falafels) sont de loin les supports iconographiques les plus mobilisés pour évoquer un multiculturalisme qui juxtapose les représentations symboliques de 
M. Mar Seck, Sénégalais, importateur depuis plus de vingt-cinq ans de masques et objets d'artisanat africains dans son magasin Darou Salam, rue Labat (Paris XVIII'). Dans les cultures africaines, la sculpture des masques est une dominante majeure de l'art plastique, toutefois les masques sculptés ne sont pas conçus pour être contemplés comme œuvres d'art, mais pour être utilisés à l'occasion de cérémonies rituelles sociales ou religieuses. @ Gilles Crampes

communautés dont les pratiques semblent déterritorialisées et anhistoriques. Les images qui ponctuent les discours touristiques sont aussi celles des corps considérés comme exotiques ${ }^{10}$, comme ces rares images d'hommes noirs ou de femmes voilées dans le Lonely Planet, mises en contexte multiculturel sur le marché de Belleville. Cette évocation des immigrés renvoie parfois aux clichés les plus basiques. À propos du passage Brady, les rédacteurs du Guide du Routard disent aimer "croiser le sourire de ces Indiens qui tiennent les restaurants et épiceries". En ce qui concerne la communauté turque, ils précisent carrément au lecteur: "Grosses moustaches et

kebabs sont de mise jusque dans la rue des Petites-Écuries, avec ses commerces, restos et autres boutiques qui rappellent les bazars d'Istanbul ${ }^{11}$."

La concentration de telles traces et ambiances matérialise l'ancrage local de groupes qui participent à des circulations globales, produisant des ethnoscapes. Ces paysages migratoires - Chinatown, Little India, Little Africa, etc. - sont monothématisés et renvoient à une figure migratoire préférentielle qui tait la multiplicité possible des provenances et appartenances (le Chinatown parisien renvoie indifféremment à une immigration chinoise, asiatique ou indo-chinoise). Les marqueurs explicites typifient les paysages urbains en les associant à une provenance en décalage avec le peuplement effectif. Ainsi, les enseignes Pondichéry ou Taj Mahal construisent le passage Brady 
comme un passage gastronomique indien, en dépit d'un entrepreneuriat pakistanais qui y est devenu majoritaire. Cette simplification rappelle celles opérées à New York pour Little Italy ${ }^{12}$ ou Chinatown ${ }^{13}$, ou dans le Little India de Singapour ${ }^{14}$.

\section{De l'exotisme au cosmopolitisme}

Ces ethnoscapes sont présentés comme des subversions de l'ordre spatial parisien supposées transporter le visiteur vers d'autres contrées: "Déambuler à travers le passage Brady, c'est presque s'aventurer dans une contre-allée de Bombay ou Dhaka ${ }^{15}$." Cette expérience construit un rapport au lieu ambigu. Le CRT évoque un "dépaysement" : on "plonge" dans des "ambiances", avec des "faux airs" de Tunis ou de Ouagadougou. C'est la distance par rapport à Paris qui fonde le rapport au lieu, construisant une altérité au cœur de la destination parisienne, sous la forme du double voyage. Le quartier, présenté comme "ethnique", constitue un Ailleurs tout comme l'immigré constitue un Autre qui n'est pas assimilable à la figure normative du Parisien, mais ajoute une touche d'exotisme à la destination.

Cependant, l'expérience migratoire métropolitaine dépasse la mise en contact avec un Ailleurs ethnicisé et relocalisé. Elle s'inscrit dans une multiplicité d'expériences possibles, entre multiculturalisme et cosmopolitisme. La juxtaposition de ces paysages produit la ville multiculturelle, un patchwork au sein duquel des groupes spécifiques sont "en situation de co-présence ${ }^{16 "}$. Le CRT décline cette thématique en parlant du monde qui "se donne rendez-vous à Paris", avec quelques localisations privilégiées: "l'Inde à La Chapelle, l'Afrique à Château-Rouge, l'Asie à Belleville et dans le Triangle d'or". Dans certains quartiers parisiens, la mise en récit transcende le pat- chwork multiculturel et adopte une perspective cosmopolite, un registre qui englobe les différences dans une culture urbaine globalisée ${ }^{17}$. C'est le cas de Belleville, qui symbolise ce métissage localisé de la ville-monde : "L'air est saturé d'arômes de coriandre, de safran et de cumin et des sonorités exotiques des langues africaines et asiatiques ${ }^{18}$." L'Office du tourisme parisien promeut également une expérience particulière de la ville, en proposant une promenade dans le "Paris cosmopolite", en éditant un guide gastronomique qui invite à un "tour du monde des saveurs" ou en suggérant dans son Paris Shopping Book un itinéraire de shopping pour les "Ethic Ethnics". Ainsi, la communication touristique se
La découverte touristique de l'immigration a ses hauts lieux institutionnels : l'Institut du monde arabe, la Grande Mosquée de Paris ou le Musée de l'histoire de l'immigration. contente de récits enchantés et exotisants pour donner à voir la dimension multiculturelle ou cosmopolite de la destination parisienne, sans renouveler en profondeur les imaginaires associés à Paris. Au-delà de cette communication, comment s'opèrent la production matérielle des ethnoscapes et leur mise en tourisme?

\section{Des communautés migratoires actrices de la mise en tourisme}

La découverte touristique de l'immigration a ses hauts lieux institutionnels : l'Institut du monde arabe, la Grande Mosquée de Paris ou le Musée de l'histoire de l'immigration. Ces lieux sont les "marques ${ }^{19}$ ", explicites et institutionnalisées, de l'histoire migratoire française et parisienne, permettant un discours ou une sémiotique contrôlés par l'agencement d'une collection et d'une muséo- 
graphie $^{20}$. En réalité, la mise en tourisme des espaces migratoires est polymorphe et ouverte à des significations plurielles. Dans quelle mesure les communautés concernées jouent-elles un rôle dans la mise en récit du Paris migratoire à destination des touristes?

Dans des pays comme le Royaume-Uni, l'Australie ou le Canada, où le multiculturalisme correspond à une modalité politique de gestion de la diversité, les lieux de concentration des commerces ethniques, notamment asiatiques, constituent les portes d'entrée symboliques des diasporas dans la ville. Ils font l'objet d'une importante mise en tourisme ${ }^{21}$. Celleci est souvent perçue comme extérieure, résultant de politiques de régénération urbaine mâtinées d'objectifs culturels, comme pour l'East End londonien $^{22}$ ou le Chinatown de Sydney ${ }^{23}$.

Toutefois, les transformations de ces quartiers ne résultent pas seulement de discours et de politiques exogènes et exotisants, elles reposent aussi, dans la plupart des cas, sur les efforts des entrepreneurs issus des minorités ethniques concernées. Dixon Street Chinese Committee à Melbourne ${ }^{24}$, Balti Triangle Association à Birmingham ${ }^{25}$, Little Havana Merchants Association ${ }^{26}$ Little Italy Restoration Association à New York ${ }^{27}$ sont autant d'organisations créées par des immigrés qui se sont alliés aux politiques publiques de régénération urbaine. Elles accompagnent l'évolution des paysages fortement exotisés, notamment des enseignes ou de la décoration des restaurants ${ }^{28}$, pour plaire à un public très large, en particulier parmi les touristes internationaux occidentaux. Ces initiatives sont inégalement acceptées au sein des quartiers d'immigration et peuvent provoquer des débats internes, comme pour les projets de mise en tourisme du Chinatown de New York ${ }^{29}$.
Les immigrés ne sont pas seulement les sujets d'une mise en scène touristique : certains entrepreneurs prennent part au processus d'auto-exotisation dans le cadre d'alliances avec les acteurs urbains.

\section{Chinagora, auto-exotisation et circulation de capitaux}

L'évolution du complexe Chinagora illustre la participation des entrepreneurs et des capitaux chinois dans la construction d'un imaginaire exotique de la Chine, ainsi que la complexité des circulations transnationales, entre tourisme et migrations. Chinagora est un ensemble hôtelier situé à Alfortville, dans la première couronne francilienne, à la confluence de la Seine et de la Marne, dans une zone dénuée d'attractions touristiques et largement marquée par les fonctions industrielles. Construit dans les années 1980, propriété d'une entreprise chinoise d'État, il a une réelle portée géostratégique : il symbolise l'ouverture de la Chine à l'économie de marché. Le bâtiment est conçu comme une immense pagode par un architecte chinois.

Tous les codes de l'exotisme sont présents : toits incurvés, couleurs vives, ornements ouvragés (voir photo ci-contre).

Le complexe comporte alors un hôtel, un centre de congrès et une galerie de commerces ethniques. Mais le lieu ne réussit jamais à décoller et fait faillite en 1997. Il est repris en 2003 par le groupe Nouveau Monde, dont l'un des dirigeants, français d'origine chinoise, affirme à la presse que son appartenance à la diaspora lui donne un avantage significatif pour faire du complexe un lieu central, qu'il veut à la fois diasporique, commercial et touristique.

20. Fabrice Grognet, “Quand l'étranger' devient patrimoine français. Les collections ethnographiques à inventer du musée de la CNHI", in Hommes \& Migrations, $n^{\circ}$ 1267, 2007, pp. 28-37. 21. Joseph Conforti, op. cit. ; Michael Hall, Jan Rath, "Tourism, migration and place advantage in the global cultural economy", in Jan Rath (dir.), Tourism, Ethnic Diversity and the City, Londres, Routledge, 2007, pp. 1-24.

22. Stephen Shaw, Susan Bagwell, Joanna Karmowska, "Ethnoscapes as spectacle: reimaging multicultural districts as new destinations for leisure and tourism consumption", in Urban Studies, vol. 41, n 10, 2004, pp. 1983-2000. 23. Anna-Lisa Mak, "Negotiating identity: ethnicity, tourism and Chinatown", in Journal of Australian Studies, vol. 27, n 77, 2003, pp. 93-100. 24. Ibid. 25. Trevor Jones, Monder Ram, "Urban boosterism, tourism and ethnic minority enterprise in Birmingham", in Jan Rath (dir.), op. cit., pp. 50-66. 26. Gaston Alonso, "Selling Miami: tourism promotion and immigrant neighbourhoods in the capital of Latin America", in Jan Rath (dir.), op. cit., pp. 164-180. 27. Joseph Conforti, op. cit. 28. C'est-à-dire conçus pour correspondre à un imaginaire exotique. Voir Jean-François Staszak, op. cit. 
Le projet connaît aussi des difficultés. En 2011, le groupe hôtelier chinois Huatian rachète le site pour le transformer en hôtel de luxe. Les dirigeants sont chinois, ils font venir de Chine des cuisiniers censés apporter un gage d'authenticité au restaurant, les Chinois résidant à Paris constituent la majeure partie du personnel de l'hôtel. Pourtant, l'exotisme reste de mise, d'immenses masques "traditionnels" ont été ajoutés sur les murs du bâtiment.

\section{Promenades urbaines et mise en récit des expériences migratoires}

Il existe des modalités alternatives de mise en tourisme des quartiers d'immigration par des circuits culturels ou mémoriels. Ces circuits révèlent une pluralité de formes (des balades urbaines aux découvertes gastronomiques), des identités migratoires signifiées (chinoise, indienne, africaine), des échelles de la mise en tourisme (du bâtiment au quartier, voire au parcours urbain) et des expériences engagées (du festif au didactique). Plusieurs initiatives associatives sont nées ces dernières années, avec la création de balades dans les quartiers d'immigration à Paris (notamment dans les XVIII et XX ${ }^{e}$ arrondissements) ou en banlieue, généralement organisées autour de la découverte d'une communauté migratoire : les Maliens dans le XVIII ou à Montreuil, les Indiens au sens large autour de la gare de l'Est, de la gare du Nord ou à La Courneuve.

Belleville, poste avancé du front de gentrification de l'Est parisien, associé à l'imaginaire du Paris populaire et cosmopolite, occupe une place particulière dans l'histoire de ce mouvement. Plusieurs associations y proposent un tourisme "participatif". Les balades sont ponctuées par des rencontres avec des habitants, témoins de l'authenticité de ces espaces urbains ordinaires, et suggèrent un contrepoint idéologique à l'offre touristique perçue comme bourgeoise et sclérosée du Paris monumental. Sophie Corbillé a analysé les mécanismes sur lesquels se construit "lenchantement de la diversité" via les visites menées par Belleville insolite, association créée en $1999^{30}$, par la sublimation de la figure classée et ethnicisée de l'indigène. Qu'il symbolise dans cet espace traversé de dynamiques sociales com-

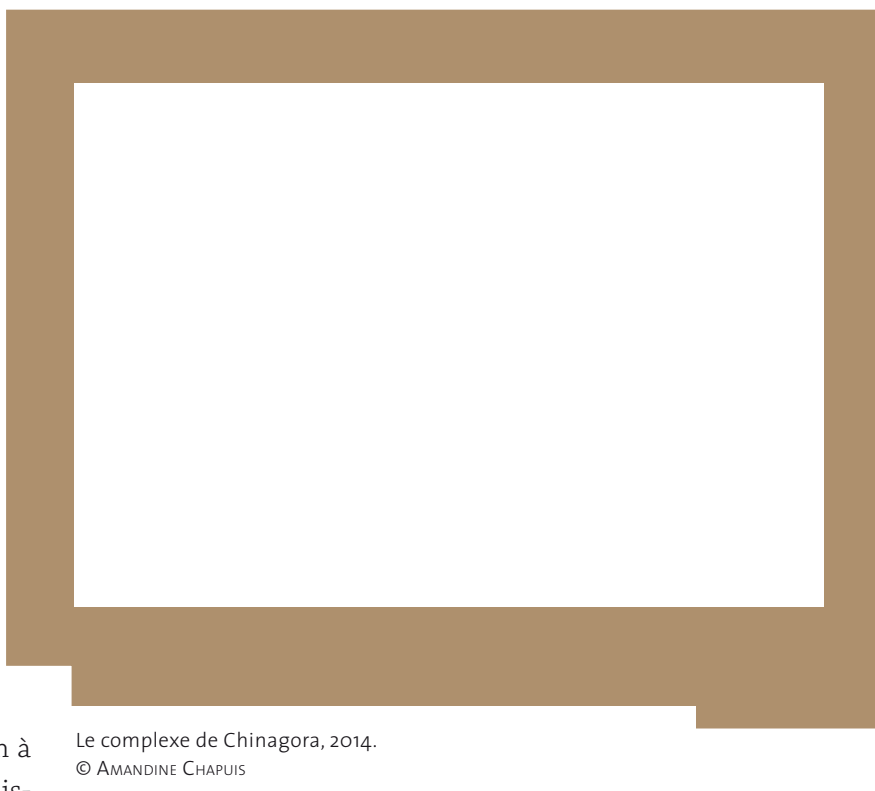

plexes l'immigré, l'ouvrier ou encore l'artiste, "cet être réduit à une culture et assigné à une identité est ce qui reste après la soustraction des rapports socio-historiques dans lesquels l'individu est inséré (...) [et] constitue un élément clé du dispositif touristique ${ }^{31 "}$. Forgés à l'aune de ces valeurs, de jeunes militants et diplômés ont poursuivi ces initiatives et intégré les collectivités territoriales, de sorte que cette approche différente de celle de l'industrie touristique classique s'est diffusée au sein des Comités départementaux du tourisme de Seine-Saint-Denis et du 
Val-de-Marne (CDT 93 et 94) ou des organismes patrimoniaux tel le Conseil de l'architecture, de l'urbanisme et de l'environnement (CAUE) du Val-deMarne, qui a monté une balade sur les traces des migrants italiens de Nogent-sur-Marne.

Témoin de ce lien fort entre caractéristiques sociales des quartiers, approche touristique et acteurs, le CDT 93 développe la thématique de la diversité ethnique comme moteur de l'économie touristique de ses territoires. Il encourage et commercialise un grand nombre de ces balades, y compris celles concernant des arrondissements du Nord-Est parisien, se substituant aux acteurs institutionnels du tourisme parisien moins mobilisés.

\section{Dire des parcours personnels}

Que nous disent ces initiatives du rapport entre tourisme et migrations ? Par-delà la mise en avant des ambiances et l'idéalisation du cosmopolitisme, ces balades sont confrontées à la question de la spécificité de chaque migration face au syncrétisme d'une possible condition migratoire ou de la relation des migrants à la société Il existe des parisienne ou au quartier.

modalités alternatives

de mise en tourisme

des quartiers d'immigration

par des circuits culturels

ou mémoriels.
Cela se lit dans les modalités des balades, qui peuvent opérer par spécialisation sur une origine migratoire donnée - le sous-continent indien pour l'association Anardana, ou le Paris algérien pour APCV (Agence de promotion des cultures et du voyage) - ou bien insister sur la dimension cosmopolite d'un quartier, comme Belleville. Mais le statut et le référentiel de ce qui est présenté n'apparaissent pas figés : la balade dans le "Petit-Maghreb" à Barbès-Goutted'Or par APCV va de l'Algérie à Paris, des sanspapiers aux mouvements locaux de résistance à la gentrification. La présentation par Anardana est traversée par la diversité des échelles d’appréhen- sion du phénomène migratoire (l'Indien devient une synecdoque d'origines diverses, du Pakistan au Népal) et joue sur les représentations des pratiques migratoires tout en les déconstruisant. Contre les assignations géographiques du marketing territorial qui renvoient tel lieu au Caire ou à Ouagadougou, ces balades véhiculent et signifient des échelles et appartenances bien plus complexes, qui s'expliquent aussi par les parcours de vie de leurs initiateurs.

Dans leur conception et leur réalisation, ces balades impliquent soit directement des migrants, soit des individus dans une position de passeurs culturels. Les guides de Baština ${ }^{32}$ sont des migrants eux-mêmes, ce que revendique son fondateur Stefan Buljat, qui s'est rapproché d'une association de jeunes Maliens pour monter son activité : “L'idée, c'est que des gens qui sont issus de ces parcours migratoires puissent faire découvrir leur quartier, leur culture et aussi leur parcours personnel33." La fondatrice d'Anardana occupe une position d'intermédiaire. Elle est mariée à un Indien, ce qui selon elle lui permet d'éviter l'écueil de l'exotisme en voyant les choses "de l'intérieur ${ }^{34 "}$ ", tandis qu'APCV est conduit par un Franco-Algérien impliqué dans les mouvements culturels et militants ${ }^{35}$.

Le point commun de toutes ces balades est la présence des migrants dans le déroulé même des circuits, qui sont ponctués par la rencontre avec des figures du quartier, notamment des commerçants (libraires, magasins alimentaires, de tissus, etc.) invités à présenter leur propre histoire. Ces balades sont donc marquées par des rencontres dédoublées : celle avec le guide, migrant ou figure de l'entre-deux faisant office de passeur, médiateur, intercesseur, et celle avec les immigrés qui font vivre les quartiers visités, commerçants, restaurateurs, artistes, acteurs associatifs ou religieux. "Chacun a un petit rôle à jouer dans le tourisme. Le problème est de savoir si les migrants ont les rôles principaux ou jouent les porte-hallebardes à l'arrière-plan", précise le fondateur de Baština. 


\section{Mettre en tourisme la France postcoloniale?}

La possibilité que les migrants soient eux-mêmes acteurs de cette mise en tourisme se heurte à la réalité de la situation sociale de certains - illégalité, précarité, pauvreté - incompatible avec les préoccupations touristiques. Selon la fondatrice d'Anardana, il est parfois compliqué d'associer à ces balades certains commerçants de La Courneuve "parce qu'il y a la barrière de la langue, parce qu'il y a des histoires personnelles difficiles, parce quils sont souvent arrivés en France dans l'illégalité, ont été sans papiers, ce qui entraine un rapport aux autres, un rapport à la France particuliers".

Au-delà des situations individuelles, mettre les migrations et l'immigration en tourisme pose des questions politiques essentielles, notamment l'enjeu du public visé et de la réception de ces initiatives. Le désir de diversité plus ou moins enchantée qui s'exprime à travers la consommation touristique des quartiers migratoires peut être compris comme une forme de distinction culturelle : la fréquentation "des corps non blancs et/ou queer procure du capital culturel à ceux qui se pensent en tant que citadins cosmopolites ${ }^{36 "}$. Cependant, les visites peuvent aussi être l'occasion pour certains habitants pas forcément socialisés au cosmopolitisme d'acquérir quelques-uns des codes sans lesquels le vivre-ensemble est difficile. Sous couvert de tourisme, le public de ces balades est en grande partie constitué de Parisiens et de Franciliens.

Les expériences et les mémoires migratoires sont également plus ou moins exprimables : si l'entrepreneuriat de certaines diasporas asiatiques semble faciliter l'investissement dans la mise en scène touristique, les migrations maghrébines paraissent sous-représentées dans ce processus, ce qui peut s'expliquer de manière assez ambiguë par

la dispersion spatiale et par la profonde intégration des immigrés maghrébins et de leurs enfants à la société française, mais aussi par la violence symbolique du lien postcolonial qui prête moins à l'exotisation. Ainsi, la visite du Petit-Maghreb à Barbès-Goutte-d'Or proposée par APVC oscille entre plusieurs registres, de l'entrepreneuriat et des formes commerciales en relation avec le Maghreb aux lieux parisiens marqués par les luttes pour l'indépendance de l'Algérie et les résistances du temps présent.

Plus largement, si le modèle d'une construction nationale

Au-delà des situations individuelles, mettre les migrations et l'immigration en tourisme pose des questions politiques essentielles, notamment l'enjeu du public visé et de la réception de ces initiatives multiculturelle semble favoriser l'expression d'appartenances culturelles multiples, le modèle assimilationniste encore prôné en France n'autorise pas la revendication de la diversité, qu'elle soit culturelle, religieuse ou ethnique, au-delà de l'argument touristique et de l'exotisme lisse des restaurants, qui ne peut satisfaire une génération tiraillée entre des logiques profondément excluantes qui perdurent et des logiques culturalistes les renvoyant elles aussi à un Ailleurs éternel et mythifié.

\section{Politique(s) de l'ancrage}

Au final, la mise en tourisme des quartiers d'immigration est-elle une forme supplémentaire d'une "altérisation ${ }^{37 "}$ des individus, à travers leur exotisation ? Ou bien, et sans doute à la fois, le tourisme peut-il être considéré comme un véhicule permettant de "combler le déficit de figuration ${ }^{38 "}$ produit par un universalisme républicain invisibilisant ?L'analyse de la relation entre tourisme et migrations à Paris sert de laboratoire pour mettre en lumière un certain nombre de représentations de la participa- 
tion des populations immigrées à la vie parisienne. Elle rappelle notamment le caractère normé, voire normatif, des figures du migrant, du touriste, du Parisien. L'étude de la relation tourisme/migrations et du rôle des migrants dans l'économie politique du tourisme permet de repenser la place des uns et des autres dans la ville. Loin de constituer deux pôles hermétiques, tourisme et migrations se croisent, au-delà même de l'expérience touristique des quartiers d'immigration.

En premier lieu, l'immigration fournit une part non négligeable de la main-d'œuvre du secteur touristique, en particulier hôtelière, non seulement parce qu'il s'agit d'une main-d'œuvre facilement exploitable, mais aussi parce qu'elle dispose d'avantages non négligeables en termes de qualification (parler arabe ou chinois est aujourd'hui très recherché dans le secteur de l'hôtellerie de luxe à Paris), mais sans qu'on dispose de suffisamment de données pour comprendre comment opèrent les rapports de pouvoir au sein de ce marché du travail. Ensuite, touristes et immigrés se croisent souvent en pratique, par exemple par l'intermédiaire des activités commerciales informelles, très présentes sur les lieux les plus fréquentés comme la tour Eiffel ${ }^{39}$, et généralement pratiquées par des migrants. Enfin, les deux catégories ne s'excluent pas en termes purement analytiques : les migrants peuvent aussi être touristes eux-mêmes. Un certain nombre de visiteurs du quartier de Château-Rouge sont des migrants africains installés dans d'autres capitales européennes, qui profitent d'un séjour à Paris pour faire l'expérience de cette centralité commerciale et culturelle $e^{40}$.

Toutefois, d'un point de vue politique, touristes et migrants demeurent des figures très différentes des circulations mondiales. Le durcissement des politiques migratoires en Europe entraîne des discriminations importantes quant à l'accès des populations racialisées à l'espace touristique parisien selon leur appartenance supposée à l'une ou l'autre de ces catégories et selon leur désirabilité. Les touristes occidentaux ont sans problème "droit à Paris", les touristes chinois font l'objet d'un soin tout particulier par les autorités, destiné à garantir leur sécurité. En revanche, un fait divers de 2012 rappelle que les touristes maghrébins s'exposent à des mésaventures significatives. Au Trocadéro, une famille de Montfermeil venue faire contempler la tour Eiffel à l'oncle marocain en visite à Paris a été arrêtée en marge de manifestations. Leurs photos, sur lesquelles ils posent souriants, une glace à la main, semblent pourtant les rapprocher des représentations qu'on se fait des touristes, ce qui n'a apparemment pas été l'avis des forces de l'ordre ce jour-là ${ }^{41}$.

Par-delà ses usages à des fins de marketing urbain et les effets d'euphémisation des relations de classe et de race ${ }^{42}$, les différents enjeux soulevés par le tourisme du fait migratoire - multiculturalisme, cosmopolitisme, rapport au territoire - impliquent un questionnement plus fondamental sur les formes de l'ancrage et du vivre-ensemble des citadins qui composent la métropole parisienne. 